\title{
DECOLONIZE THE LIBRARY - BERICHT ÜBER DIE ONLINE-VERANSTALTUNG (27. JÄNNER 2021)
}

\section{von Sandra Sparber}

Zusammenfassung: Der vorliegende Bericht schildert die Veranstaltung „Decolonize the Library“, welche koloniale Repräsentationen in Bibliotheksbeständen und -praktiken zum Inhalt hatte.

Schlagworte: Wissensordnung; Bibliotheksbestand; Dekolonisierung; Rassismus

\section{DECOLONIZE THE LIBRARY - REPORT ON THE ONLINE EVENT (27 JANUARY 2021)}

Abstract: This report describes the event "Decolonize the Library", which focused on colonial representations in library collections and practices.

Keywords: Knowledge system; Library collection; Decolonization; Racism

DOI: https://doi.org/10.31263/voebm.v74i1.6851

(c) Sandra Sparber

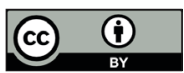

Dieses Werk ist - exkl. einzelner Logos und Abbildungen - lizenziert unter einer Creative-Commons-Lizenz Namensnennung 4.0 International-Lizenz 
Nutzer*innen von Bibliotheken sind an allen Ecken und Enden mit kolonialen Fortschreibungen konfrontiert. Wir finden sie etwa in unseren Wissensordnungen, in Schlagworten, in unseren - nicht nur historischen - Beständen, in unseren Erwerbungspraktiken und unserer Personalpolitik.

Um auch im deutschsprachigen Bibliothekswesen auf diese Missstände aufmerksam zu machen sowie um gemeinsam Möglichkeiten und Methoden zur Überwindung dieser Praktiken, die sich in unseren Berufsalltag eingeschrieben haben, zu finden und zu entwickeln, lud die c3.Bibliothek für Entwicklungspolitik zu einem Austauschtreffen. Moderiert von Gabi Slezak wurden einführend Erfahrungen aus der Praxis im Umgang mit sogenannten problematischen Medien reflektiert. Dani Baumgartner von der c3.bibliothek berichtete im ersten von insgesamt vier Vorträgen von Erfahrungen mit Leser*innen, die das Bibliotheksteam auf rassistische Inhalte in den Werken eines Sonderbestandes aufmerksam machten. Sie schilderte die Ambivalenz hinsichtlich der Bedeutung dieser Werke, die einerseits von Rassismen durchzogen sind und andererseits als Quellenmaterial der Forschung zur Verfügung stehen sollten. Zudem verwies sie auf den institutionsinternen Konsens, Räume frei von Rassismus zu halten. Unerwähnt blieb, ob und gegebenenfalls welche konkreten Schritte gesetzt wurden, um diesem Anspruch gerecht zu werden.

Doch was bedeutet die Bezeichnung Kolonialität überhaupt? Hier lieferte Nora Schmidt von der ub.mdw eine begriffliche Annäherung. Sie versteht darunter privilegierte Vorrechte des globalen Nordens, die zu Ungleichheit und Ungerechtigkeit führen. Diese haben sich auch im Wissenschaftssystem etabliert und sind u.a. bestimmend für die Durchlässigkeit von Publikationsmedien. Dass Medien des globalen Südens beim Bestandsaufbau unberücksichtigt bleiben, werde durch die Kommerzialisierung von Open Access, die Quantifizierung wissenschaftlicher Kommunikation in Form von Bibliometrie und Szientometrie und durch predatory publishing gefördert. Bibliotheken unterstützten diese Entwicklungen insbesondere durch den Ankauf von Paketen; ein Umstand der einander immer ähnlicher werdende Bestände zur Folge habe. Aufgebrochen werden könne dies nur, wenn sich Bibliotheken bzw. die handelnden Personen ihrer Privilegien bewusst werden. Somit berühre die Problematik auch berufsethische Aspekte, wenn wir uns etwa zwischen dem Weg des geringeren Widerstands und alternativen Möglichkeiten entscheiden müssen. 
Wie und anhand welcher Kriterien der Ankauf von Kinderbüchern bei BAOBAB, dem Verein für globales Lernen, Sprachförderung und Diversität, erfolgt, berichtete Magdalena Emprechtinger. Basierend auf dem Ansatz der vorurteilsbewussten Bildung, erläuterte sie die wichtigsten inhaltlichen Aspekte der Vorauswahl. Dazu zählen:

1. Vielfalt in der Darstellung, wie etwa unterschiedliche Erfahrungen und Familienstrukturen, Sprache, Geschlechterrollen und sozioökonomische Hintergründe

2. Gleichwertigkeit statt Paternalismus, wobei insbesondere die Rolle der Protagonist*innen, die nicht der Mehrheitsgesellschaft angehören und die Frage danach, wie Angehörige von Minderheiten ihre Probleme lösen, im Fokus stehen sollen

3. keine Stereotype und/oder diskriminierenden Inhalte in Illustrationen und Sprache

4. Beispiele, wie man sich gegen Diskriminierung wehren kann

Die Vortragende merkte an, dass Bücher, die diesen Kriterien nicht entsprechen, auch an Verlage zurückgesendet werden. Ihr Bericht zeigte sehr deutlich, dass reflektierte Medienauswahl entsprechende Kompetenzen und Zeitressourcen benötigt. Für die Auswahl von Kinderbüchern stellte die Kollegin gleichzeitig eine solide, praxistaugliche und leicht integrierbare Orientierungshilfe zur Verfügung.

Sarah Schmelzer von der c3.Bibliothek konzentrierte sich in ihrem Vortrag auf die praktische Arbeit in Zusammenhang mit rassistischen Inhalten in den Bibliotheksbeständen. Sie sieht sich mit ihrer Einrichtung am Anfang einer Diskussion, die intern geprägt ist von begrenzten Ressourcen und Tätigkeiten, die nebenbei passieren. Das Wichtigste sei die dialogische Herangehensweise, da der gesamte Prozess als solcher ein wichtiges Ergebnis darstelle. Das Wegsperren von Medien oder einzelner Titel stellt für sie keine Option dar, wenngleich sie erwähnte, einzelne rassistische Werke entsorgt zu haben. Auch das Kennzeichen problematischer Bücher sei für sie nicht zielführend. Als wissenschaftliche Bibliothek stehe der Sammelauftrag im Zentrum der Überlegungen.

Jana Rumler aus dem Museum für Naturkunde Berlin stellte im Anschluss an die Kurzreferate als Mitherausgeberin der Open Access-Zeitschrift LIBREAS. Library Ideas den Call for Papers für die Ausgabe 39 mit dem Schwerpunkt „Antirassistisch und/oder kolonial? Bibliotheken im Spannungsfeld antirassistischer und kritischer Auseinandersetzung mit dem eigenen kolonialen Erbe“ vor. Kolleg*innen aus den Gedächt- 
niseinrichtungen sind eingeladen, ihre Inhalte bis zum 30. Juni 2021 einzureichen und sich an der aktuellen Debatte zu beteiligen.

An der Online-Veranstaltung bestand mit der ausgeschöpften Onlinelizenz-Kapazität von hundert Teilnehmer*innen reges Interesse. Mehrere Kolleg*innen äußerten den Wunsch, sich in die Debatte einzubringen und ein weiteres Austauschformat zum Thema zu organisieren. Es ist dringend an der Zeit, dass wir uns mit den Strukturen und Praktiken in unseren Bibliotheken auseinandersetzen und unseren Teil dazu beitragen, diese weitgehend diskriminierungsfrei zu gestalten.

Sandra Sparber, M.A. Sigmund Freud Museum, Bibliothek E-Mail: s.sparber@freud-museum.at

\section{Literatur}

Decolonize the Library. Online Austauschtreffen (Videoaufzeichnung der Veranstaltung vom 27. Jänner 2021): https://youtu.be/T7gCyHcQucs LIBREAS. Library Ideas, Call for Papers mit dem Schwerpunkt „Antirassistisch und/oder kolonial? Bibliotheken im Spannungsfeld antirassistischer und kritischer Auseinandersetzung mit dem eigenen kolonialen Erbe“: https://libreas.wordpress.com/category/libreascall-for-papers/

Nora Schmidt (2020). The Privilege to Select. Global Research System, European Academic Library Collections, and Decolonisation (Version Compressed). Lund University, Faculties of Humanities and Theology, Lund Studies in Arts and Cultural Sciences, Lund, Sweden. https://doi.org/10.5281/zenodo.4302687 